\title{
DPIV and interferometry technique for velocity field and concentration fields measurement in crystal growth
}

\author{
Q. Kang $\square$ L. Duan ${ }^{* *}$ \\ National Microgravity Laboratory/ CAS; Institute of Mechanics, CAS \\ Beijing, 100080 China
}

\begin{abstract}
The property of crystal depends seriously on the solution concentration distribution near the growth surface of a crystal. However, the concentration distributions are affected by the diffusion and convection of the solution. In the present experiment, the two methods of optical measurement are used to obtained velocity field and concentration field of $\mathrm{NaClO}_{3}$ solution. The convection patterns in sodium chlorate $\left(\mathrm{NaClO}_{3}\right)$ crystal growth are measured by Digital Particle image Velocimetry (DPIV) technology. The 2-dimentional velocity distributions in the solution of $\mathrm{NaClO}_{3}$ are obtained from experiments. And concentration field are obtained by a Mach-Zehnder interferometer with a phase shift servo system. Interference patterns were recorded directly by a computer via a CCD camera. The evolution of velocity field and concentration field from dissolution to crystallization are visualized clearly. The structures of velocity fields were compared with that of concentration field.
\end{abstract}

Keywords: PIV; Interferometry, Crystal growth; Buoyancy convection

\section{INTRODUCTION}

On the ground, the convections caused by buoyancy are generally unavoidable in the crystal growth process from solution. Firstly, a concentration field is a direct factor to the effect of crystal growth. On the other hand, spatial variety of concentration field could induce the convection of solution outside the crystal due to the buoyancy effect. Furthermore, the convection of solution could change the spatial distribution of concentration. The interaction of concentration field and velocity field is a complex problem. Therefore, it is significant to obtain the velocity field and concentration field in crystal growth process to understand both of the growth rate of crystal and the quality of crystal.

The optical technique that is non-destructive and for whole flow field has been widely used to study the process of the crystal growth experimentally. However, most works given only simple character parameters and a few qualitative analyses results [1-3], such as the thickness of the diffusion boundary layer and the possibility of buoyancy convection. The information deals with the whole field concentration distribution and velocity distribution together with the process of the crystal growth and the fluid dynamics idea are few experimentally.

\section{DIAGNOSTIC METHOD}

In the present experiment, the crystal growth cell is a container with rectangular cross-section of $20.0 \mathrm{u} 12.0 \mathrm{~mm} 2 \mathrm{and}$ $14.0 \mathrm{~mm}$ high. The lateral walls of the container consist of transparent K9 optical glass for optical interferometer measurement and PIV application.

～kq@imech.ac.cn; phone +86 10 6262-6158; fax +86 10 6261-5524; http://www.imech.ac.cn, http://nmlc.imech.ac.cn; National Microgravity Laboratory, Institute of Mechanics, No 15 Zhong Guan Cun Road, Beijing 100080, China;

** duanli@imech.ac.cn; phone +86-10-6261-5536; fax +86-10-6261-5524; http://www.imech.ac.cn, http://nmlc.imech.ac.cn;

National Microgravity Laboratory, Institute of Mechanics, No 15 Zhong Guan Cun Road, Beijing 100080, China 


\subsection{Laser interferometry for concentration field}

In order to obtain the concentration distribution of solution, a Mach-Zehnder micro-interferometer with a phase shift system and an image system were used in the experiments as shown in Fig. 1. He-Ne laser, $\mathrm{L}_{1}, \mathrm{~L}_{2}, \mathrm{M}_{1}, \mathrm{M}_{2}, \mathrm{M}_{3}, \mathrm{Bs}_{1}$ and $\mathrm{Bs}_{2}$ compose a typical Mach-Zehnder interferometer. The mirror $\mathrm{M}_{3}$ could be driven by a Piezoelectric Translator (PZT) for a phase shift of interferograms. The PZT controller was connected with the computer of an image system. The interferograms were magnified by Lens $\mathrm{L}_{3}, \mathrm{~L}_{4}$, and were recorded by the image system, which was synchronized with the phase shift system of the interferometer. The four-steps phase shift technique was used, that is, four images of the interference fringe must be acquired to invert fringe image into refractive index field at each state. The interferometry theory and experiment method have been introduced by Duan[4]. Refractive index distributions were calculated by using a phase-shift technology and a method of phase unwrapping. The concentration distribution is obtained by using the relationship between the concentration and the refractive index. Depending on the study of the interference pattern, not only the concentration distributions could be obtained, but also the convection phenomena and the modality of the convection pattern could be estimated in the process of $\mathrm{NaClO}_{3}$ crystal growth.

The relation between refractive index and concentration of $\mathrm{NaClO}_{3}$ solution is an essential parameter to invert the interferometry image to concentration distribution, and was measured by a refractive meter (Type: WAY-15 ABBE REFRACTOMETER). The results of measurements are listed in Table 1. The temperature of the $\mathrm{NaClO}_{3}$ solution was fixed at $20.09 \mathrm{C}$ during the measurement. The refractive index of $\mathrm{NaClO}_{3}$ solution is not sensitive to the temperature change within $5 \mathrm{qC}$.

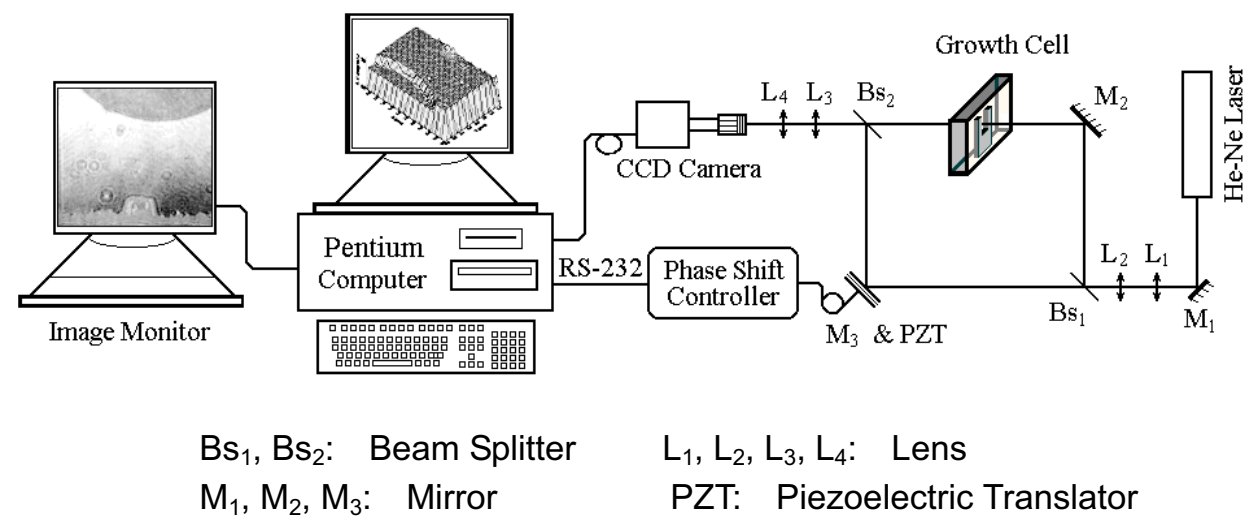

Fig. 1 Schematic diagram of interferometry system and image system

Table 1 The dependency of refractive index on concentration (for $\mathrm{NaClO}_{3}$, at 20.09C)

\begin{tabular}{|c|c|c|c|c|c|c|c|c|c|}
\hline No. & 1 & 2 & 3 & 4 & 5 & 6 & 7 & 8 & 9 \\
\hline \hline $\begin{array}{c}\text { Concentration } \\
(\mathrm{Mol} / \mathrm{L})\end{array}$ & 0.50 & 0.75 & 1.00 & 1.25 & 1.5 & 1.75 & 2.0 & 2.25 & 2.50 \\
\hline $\begin{array}{c}\text { Refractive } \\
\text { index }\end{array}$ & 1.3445 & 1.3461 & 1.3492 & 1.3512 & 1.3554 & 1.3580 & 1.3594 & 1.3631 & 1.3656 \\
\hline
\end{tabular}




\subsection{Particle Image Velocimetry for velocity field}

For the Particle Image Velocimetry (PIV), the trace particles have to be used in the fluid, and they might influence the process of the crystal growth. A system of Digital Particle Image Velocimetry (DPIV) of DANTEC measurement technology A/S with FlowMap PIV 2000 Processor was used to quantitatively measure the 2-D velocity field in a vertical cross-section of the cell during the process of the crystal growth, as shown in Figure 2. An argon ion laser was applied to illuminate the flow field. Pulse lights were shaped by an electro-optical shutter, which was controlled by a center processor with CCD camera synchronization. The resolution of CCD camera is $768 \mathrm{u} 484$ pixels with $11.6 \mathrm{u} 13.6 \mathrm{Pm}$ pixel pitch. The silver-coated hollow glass spheres of $10 \mathrm{Pm}$ in diameter as tracer particles are suspended in the $\mathrm{NaClO}_{3}$ solution. The density of the particles is $1.4 \mathrm{~g} / \mathrm{cm}^{3}$, which is close to the density of $\mathrm{NaClO}_{3}$ solution. By using a cycling lens, a $1.0 \mathrm{~mm}$ thin light sheet is shaped to illuminate a vertical cross-section at the center part of the container. This cross-correlation technique of DPIV image matching processing is used to obtain both magnitude and direction of velocity vector at the same time $[5,6]$. The cross-correlation method is much batter than the auto-correlation method. The auto-correlation generally could not determine the direction of velocity vector and zero velocity. If the method of pre-displacement is used to solve the two problems, the dynamics range of measured velocity will decrease near half. Besides, because the first exposed particle on the original image and the second exposed particle on the template image are the background noise of auto-correlation method, the accurate and success ratio of determined displacement are much less than that by using cross-correlation method, as shown in Figure 3.

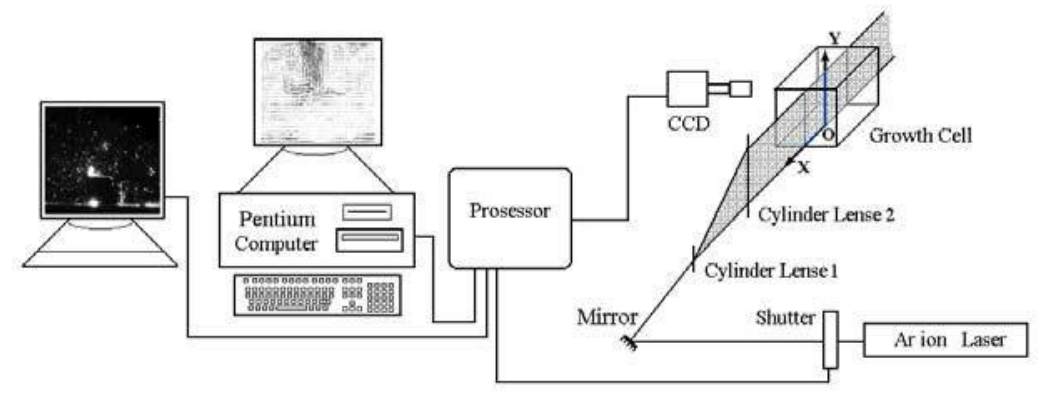

Fig. 2 Arrangement of PIV in the experiment

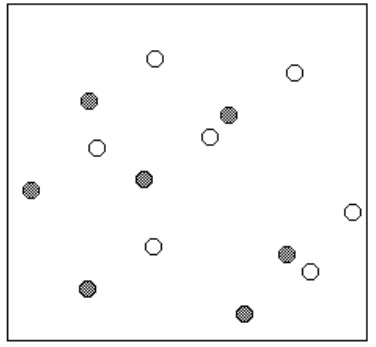

The first exposedness O The second exposedness The double exposed particle image

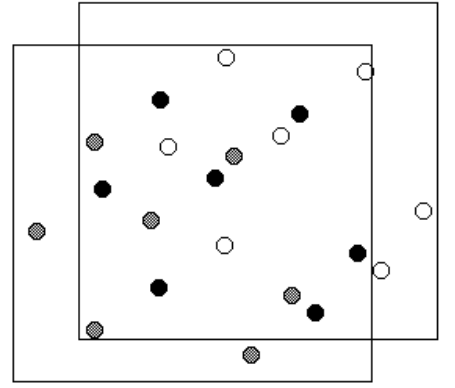

The effective particle - 0 The noneffective particle The principle of auto-correlation method

\section{(a) Auto-correlation method}

Fig. 3 The match comparison of particle image between auto-correlation and cross-correlation (continued) 


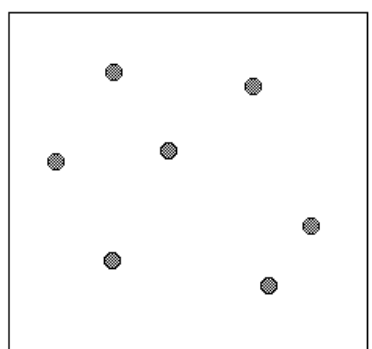

The first exposed The first particle image

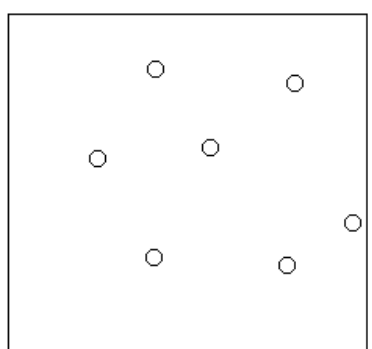

The second exposed The second particle image

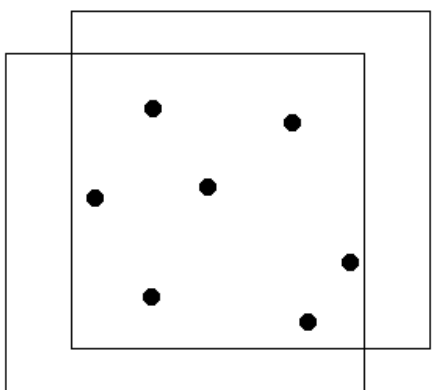

The effective particle

(b) Cross-correlation method

Fig. 3 The match comparison of particle image between auto-correlation and cross-correlation

\section{EXPERIMENTAL RESULTS WITH DISCUSSION}

A seed crystal was put into the growth cell of $\mathrm{NaClO}_{3}$ solution at $219 \mathrm{C}$ on the beginning of the experiment. The initial concentration of $\mathrm{NaClO}_{3}$ solution is $46.43 \%$, which is undersaturated solution at $21 \mathrm{QC}$. The seed crystal is dissolved at the early stage. With the dissolution of the crystal and the declines of the temperature in the solution of growth cell, the concentration of solution would reach to its saturation near $20 \mathrm{oC}$. In this case, the concentration gradient is very small in the solution around the $\mathrm{NaClO}_{3}$ crystal. It is a very short period that the crystal does not dissolve further, neither does the crystal grows in the present experiment. While the temperature of the growth cell keeps up fallen gradually, the solution would be in its super saturation state. Thus, the solution crystallizes gradually on the surface of the seeding crystal and forms concentration gradient around the crystal.

The buoyancy-driven convection in graviton field is a typical question of fluid mechanics. The asymmetry of the temperature (or/and concentration) could bring the asymmetry of the fluid density. The fluid whose density is lower would move to the direction that opposed the gravitational direction, due to the buoyancy effect. The convection will occur in the fluid if the buoyancy is large enough to overcome the viscous resistance and thermal diffusion effect (or/and concentration diffusion effect). However the convection would vanish in the space experiments. The diffusion process is a typical phenomenon of mass transfer. Pure diffusion process is very good condition to make materials, and new material will come up in the future to be prepared and tested in microgravity environment.

Figure 4 shows a particle image of flow field in the solution during the $\mathrm{NaClO}_{3}$ crystal growth. The center part on the bottom is a $\mathrm{NaClO}_{3}$ seed crystal.

Figures 5 shows a group of interferograms of the four-step phase shift. Figure 6 shows its phase map, its phase unwrapping map and its refractive index distribution. Figures 7 shows the evolution of the concentration field. In Figure 7, $\mathrm{C}$ and $\mathrm{Cf}$ are respectively the local solution concentration and the solution concentration in far field.

Figure 8 shows the evolution of the velocity field.

Figures 7(a) shows the concentration distribution at the early stage of the experiment. Because the solution is undersaturation, the seed crystal is dissolved at this stage. The concentrations of the solution around the crystal are larger than the ones in the solution far away from the crystal. Due to the existence of the density gradient caused by concentration difference between the up part of the seed crystal and two sides of the seed crystal, and the direction of the density gradient is opposite to the one of gravity, the solution around the crystal moves downward, as shown in Figure 8(a), to form a concentration buoyancy flow.

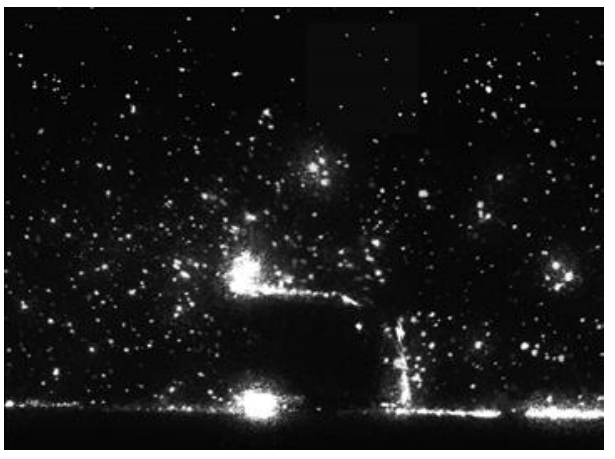

Fig. 4 A particle image of flow field 

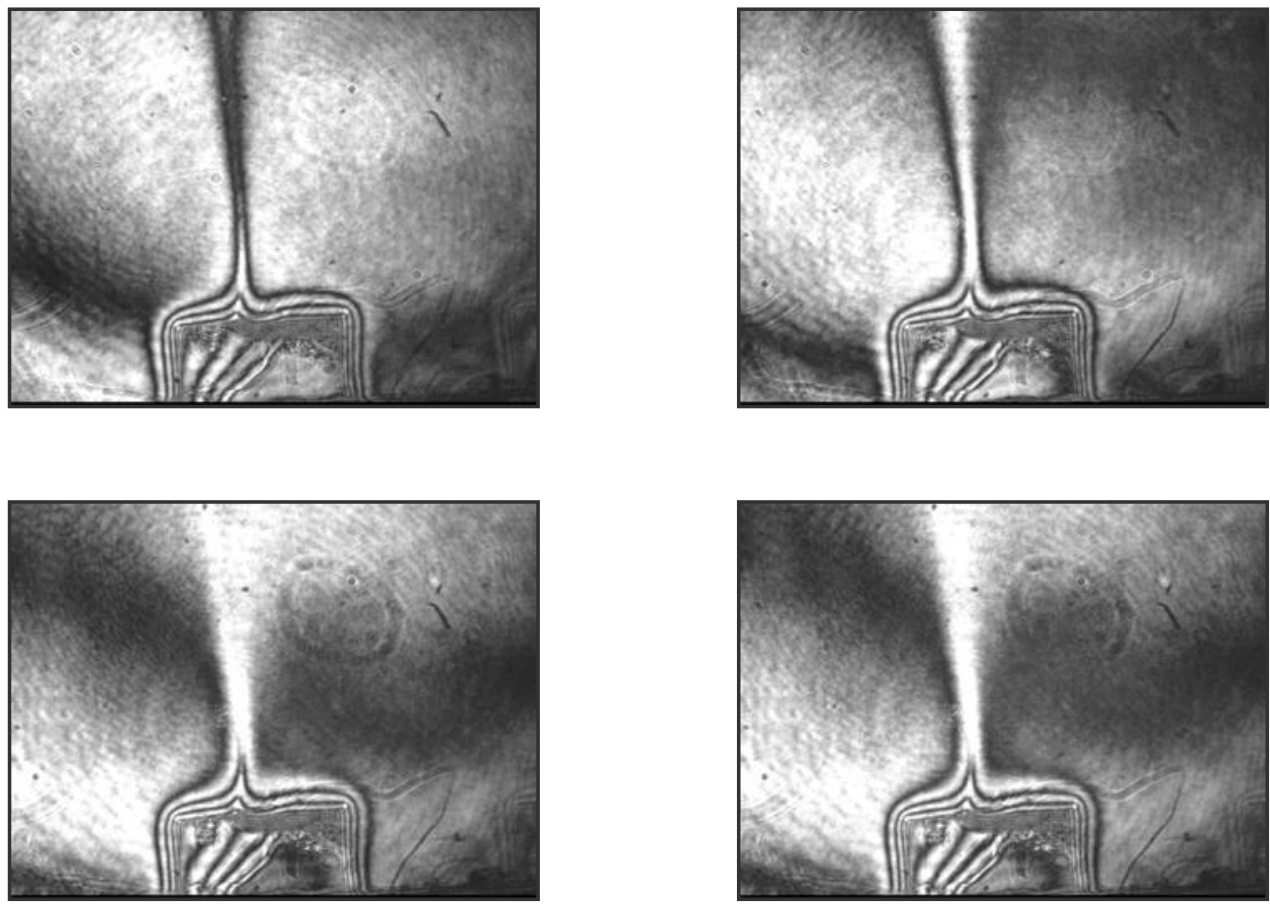

Fig. 5 The four interferograms of four-step phase shit
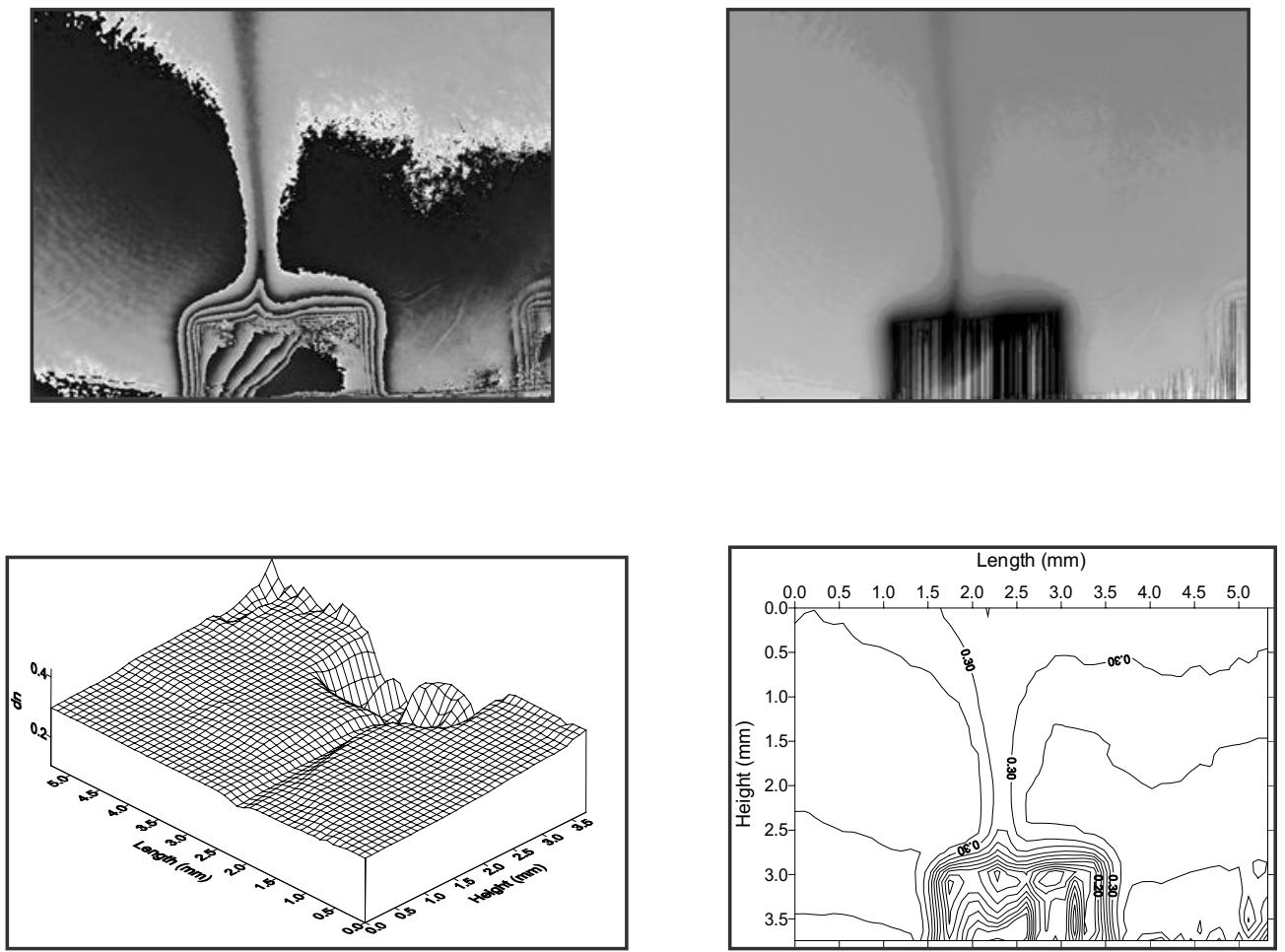

Fig. 6 A process result of refractive index field by four-step phase shift technique 


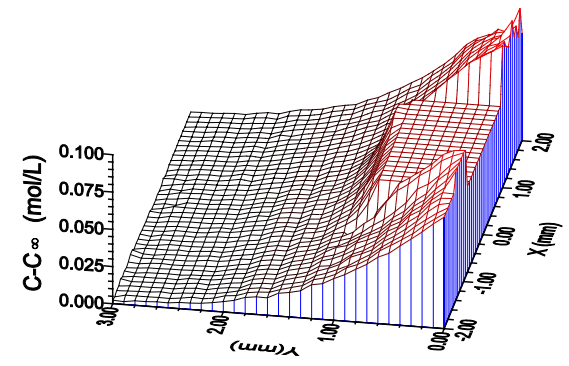

(a)

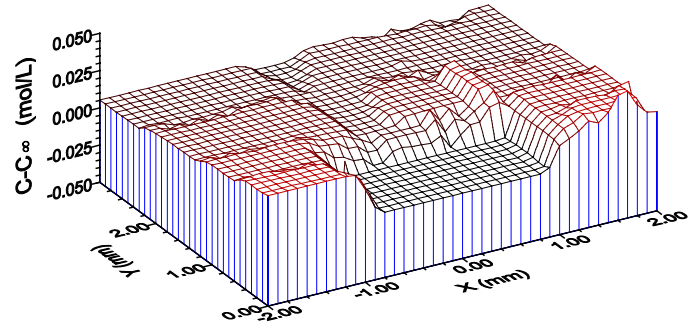

(c)

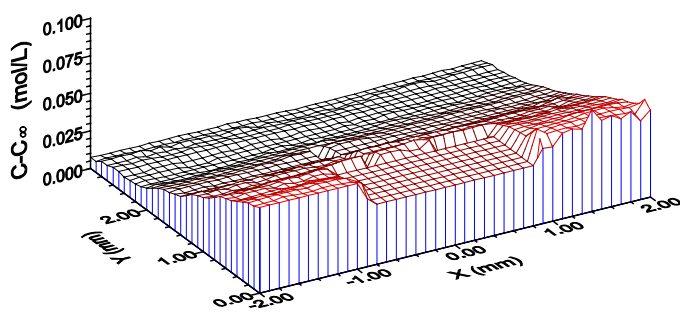

(b)

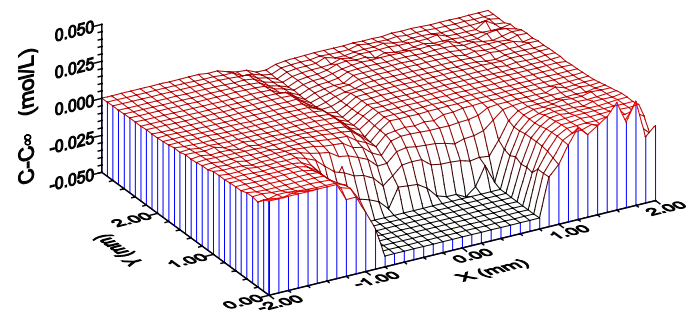

(d)

Fig. 7 The evolution of the concentration distribution

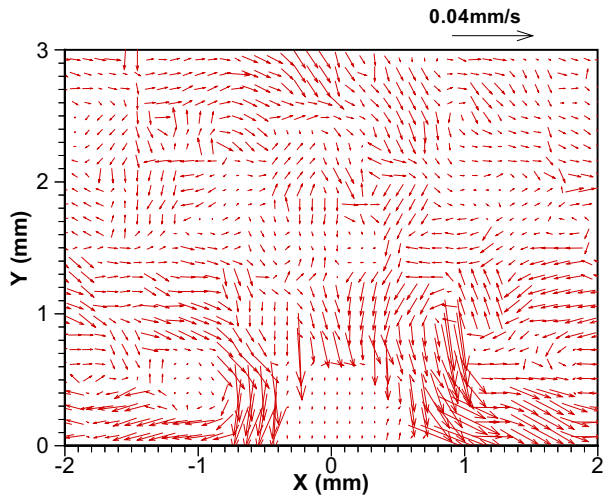

(a)

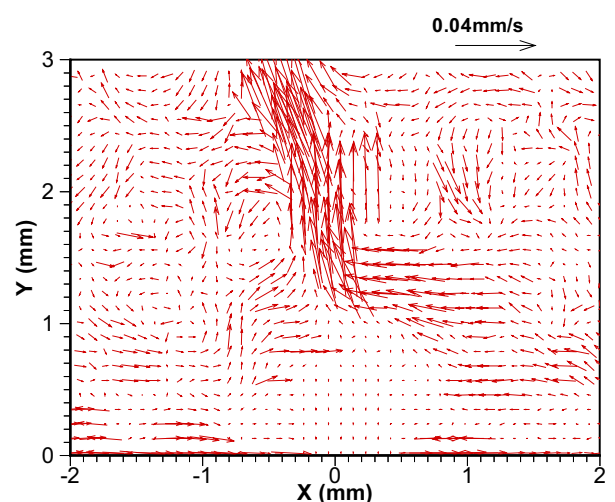

(c)

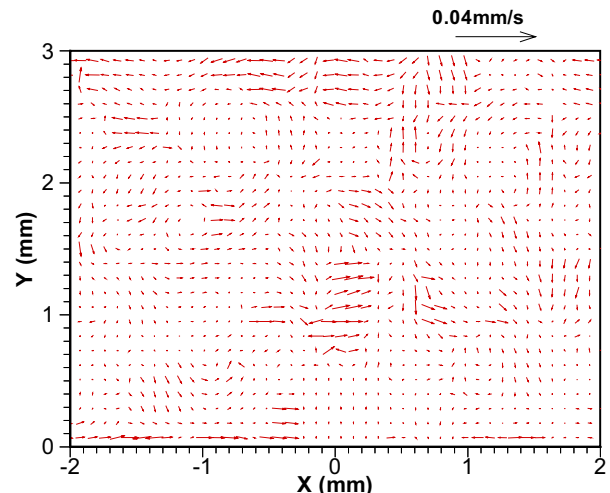

(b)

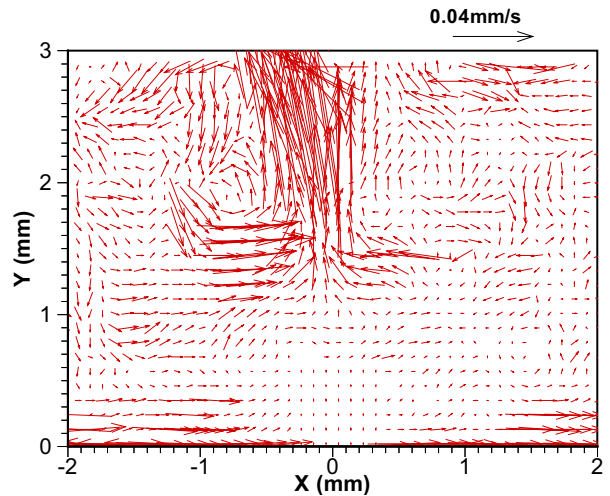

(d)

Fig. 8 The evolution of the velocity field 
Near the saturation state, the concentration gradient is very small in the solution around the $\mathrm{NaClO}_{3}$ crystal, as shown in Figures 7(b). Therefore, the convection nearly vanishes, as shown in Figure 8(b). The phenomena of concentration delamination (or gravity sedimentation) caused by gravity can be seen in Figures 7(b). It is a very short time period that the crystal does not dissolve further, and the crystal does not grow in the present experiment.

At a super saturation state, the solution crystallizes gradually on the surface of the seeding crystal and forms concentration gradient around the crystal. The concentration of the solution around the crystal is smaller than that far away from the crystal, as shown in Figures 7(c) and 7(d), and the direction of the density gradient on the top part of the crystal is directly opposite to that of gravity. Hence, the solution in the diffusion boundary layer of the crystal moves upward in the growth cell, and forms a plume flow in the upper part of the crystal, as shown in Figures 8(c) and 8(d). We can see from Figure 7(c) to Figure $7(\mathrm{~d})$ that the concentration difference around the crystal is gradually increased. The process of velocity increase can be also seen clearly in Figures 8(c)-8(d). The flow is still a buoyancy convection driven by the exchange of the concentration under the earth's gravity. The direction of the flow is opposite to that for crystal being dissolved.

These experimental results show clearly that the mass transfer is carried out mainly by the help of the convection caused by the concentration gradient during the period of the crystal growth. However, the concentration gradient would be reduced because of the convection. The coupling processes of concentration and velocity would influence the rate of the crystal growth.

\section{CONCLUSIONS}

The buoyancy convection phenomena caused by concentration asymmetry are studied in the process of crystal growth. The construction of convection pattern and the construction of concentration pattern were showed clearly. The convection phenomena that were guessed early by analyzing concentration field distribution were confirmed directly by measuring velocity field. The buoyancy convection (plume flow) during the crystal growth has been visualized.

The PIV and interferometry techniques are benefit for understanding the fluid flow phenomena of the crystal growth process and for supporting the observation of the crystal growth together with fluid process. The evolution of flow pattern and interference fringe pattern were recorded in the process of both dissolving and crystallizing. The concentration fields are obtained by a Mach-Zehnder interferometer with a phase shift system and an image system. The four-step phase shift technique is used to analyze the interferograms. The velocity fields of $\mathrm{NaClO}_{3}$ solution are also acquired successfully by a digital particle image velocimetry.

\section{ACKNOWLEDGMENTS}

This research is supported by the grant 95-yu-34 of the Ministry of Sciences and Technology of China, the grant 19789201 of the National Natural Science Foundation of China, the grant of Rector Foundation of CAS and the Foundation of China Post Doctor.

\section{REFERENCE}

1. Kazuo Onuma, Katsuo Tsukamoto and Suezou Nakadate, Application of real time phase shift interferometer to the measurement of concentration field, Journal of Crystal Growth, 129(1993) 706-718.

2. Satoru Miyashita, Hiroshi Komatsu, Yoshihisa Suzuki etl. Observation of the concentration distribution around a growing lysozyme crystal, Journal of Crystal Growth, 141 (1994) 419-424.

3. K. Onuma, K. Tsukamoto and I. Sunagawa, Role of buoyancy driven convection in aqueous solution growth; A case study of $\left(\mathrm{BaNo}_{3}\right)_{2}$ crystal, Journal of Crystal Growth, 89 (1988) 177-188.

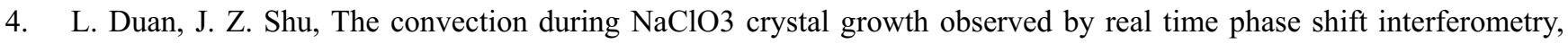
Journal of Crystal Growth, 223(2001) pp181-188.

5. Q. Kang, W.R. Hu, Studies on Bénard-Marangoni convection by PIV, 47th International Astronautical Congress, AIAA, IAF-96-J.3.10, 1996

6. FlowMap Installation \& User’s guide, DANTEC Measurement Technology A/S, Denmark, 1996 